\title{
Robust control and optimized parallel control double loop design for mobile robot
}

\author{
Ahmed J. Abougarair, Ali S. Elmolihi \\ Department of Electrical and Electronics Engineering, University of Tripoli, Libya
}

\begin{tabular}{l}
\hline \hline Article Info \\
\hline Article history: \\
Received Oct 6, 2019 \\
Revised Jan 12, 2020 \\
Accepted Mar 4, 2020 \\
\hline
\end{tabular}

\section{Keywords:}

LQR

PID

SMC

Time Delay

TWBMR

\begin{abstract}
Robots have been used in many applications in the past few decades. Moreover, due to high nonlinearity behavior of these systems, an optimal and robust control design approaches have been considered to stabilize and improve their performance and robustness. The uncertainties of the time delay on the output states of the mobile robot system have a significant influence on the system nominal performance. As a result, the work becomes here to address the influence of these uncertainties on the robot system performance. In order to achieve this objective, the nonlinear controller via sliding mode control (SMC) is designed by selecting a suitable sliding surface dynamics in which the considered robot displacement and tilt angle are sliding on. The lyapunov function is considered here to accomplish the design of the sliding control signals for robot stabilization. Furthermore, the stability of the considered system is guaranteed due to convergence of the lyapunov functions into zero when the state trajectories tend to desired set points. In addition, we consider the trajectory tracking and stabilization of TWBMR system using parallel double loop PID controllers whose controllers gains are tuning via linear quadratic regulator (LQR) approach. Finally, to demonstrate the effectiveness of SMC and PID-LQR design methods, the comparison is carried out when the nominal and uncertain conditions.
\end{abstract}

This is an open access article under the CC BY-SA license.

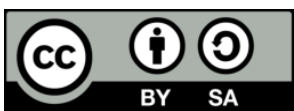

\section{Corresponding Author:}

Ahmed J. Abougarair,

Department of Electrical and Electronics Engineering,

University of Tripoli, Tripoli, Libya.

Email: a.abougarair@uot.edu.ly

\section{INTRODUCTION}

The two-wheeled model has attracted much attention in the field of control theory because they are nonlinear and under actuated with inherent unstable dynamics. Wheeled inverted pendulum is one kind of inverted pendulum system, and it names two-wheeled self-balanced mobile robot (TWBMR). The TWBMR use clean energy as a power and has advantage of small capacity, feasible rotation, which is used widely in many fields, such as traffic, survey, rescue and entertainment $[1,2]$. TWBMR is set up by cart, meanwhile, each wheel of cart is connected with an output shaft of a motor. Moreover, the motors drive the movement while maintaining self-balancing of the body of the system in the two-dimensions [3, 4]. SMC has many applications in robotics [5]. In particular, this control algorithm has been used for tracking control of unmanned surface vessels in simulated rough seas with high degree of success [6, 7]. Also, the LQR approach has been used for the design of optimal PID controllers where the controller gains are formulated as the optimal state-feedback gains, corresponding to the desired response.The time delay is a significant problem that have been interested in many previous researches as in $[8,9]$. By going in the same direction of the previous literatures for addressing the influence of time delay on the system performance, the work in this 
paper is considered here to avoid the effect of time delays on the TWBMR tracking performance is presented. This paper can be arranged as follows. In section two, the nonlinear and linear dynamic model of TWBMR is presented, in section three, the SMC design is presented, in section four PID based LQR is designed and shown, the simulation results were provided in section five and finally the conclusion is included in section six.

\section{MATHEMATICAL MODEL OF TWBMR}

The TWBMR is restricted to a plane, and it is assumed that the wheels always stay in contact with the ground and that there is no slip at the wheel's contact point. Therefore, there is no movement in the $\mathrm{z}$ axis. The set of equations of TWBMR has been developed by [6] to describe the system by the following nonlinear equations:

$$
\begin{aligned}
& \ddot{\theta}_{p}=\frac{2 k_{e} k_{m}}{R r} \gamma \dot{x}-\frac{2 k_{m}}{R} \gamma V_{a}-M_{p} g l \gamma \sin \theta_{p}-F Z \gamma \cos \theta_{p}-M_{p} l \gamma \ddot{x} \cos \theta_{p} \\
& \ddot{x}=-\frac{2 k_{e} k_{m}}{R r^{2}} \delta \dot{x}+\frac{2 k_{m}}{R r} \delta V_{a}+M_{p} l \delta \dot{\theta}_{p}^{2} \sin \theta_{p}-F \delta-M_{p} l \delta \cos \theta_{p} \ddot{\theta}_{p}
\end{aligned}
$$

Where $\gamma=\frac{1}{\left(\mathrm{I}_{\mathrm{p}}+\mathrm{M}_{\mathrm{p}} \mathrm{l}^{2}\right)}, \quad \delta=\frac{1}{\left(2 \mathrm{M}_{\mathrm{w}}+\mathrm{M}_{\mathrm{p}}+\frac{2 \mathrm{I} w}{\mathrm{r}^{2}}\right)}$

With $x$ represents the car displacement, $\theta$ is the pendulium angular displacement, $M_{p}$ is the body mass, $I_{p}$ is the body inertia, $\mathrm{K}_{\mathrm{e}}$ denotes to the back EMF, $\mathrm{M}_{\mathrm{w}}$ is the wheel mass, $\mathrm{I}_{\mathrm{w}}$ is the wheel inertia, $\mathrm{K}_{\mathrm{m}}$ is the motor torque constant, $\mathrm{R}$ is the resistance, $\mathrm{L}$ is the distance from center of gravity (COG), $\mathrm{r}$ is the wheel radius, $\mathrm{V}_{\mathrm{a}}$ is the applied voltage, $\mathrm{g}$ is the gravity, $\mathrm{F}$ is disturbance force and $\mathrm{Z}$ is the position of applied disturbance. For more details about the derivation of this model, the reader refers to [10]. These equations are then used to make a simulink model of the robot as shown in Figure 1. The linearization process is done to obtain the linearized model of the considered system for optimized PID control design. Accordingly, the state space model is derived in order to carry out the design of the LQR controller.

$$
\begin{aligned}
& {\left[\begin{array}{l}
\dot{x} \\
\ddot{x} \\
\dot{\phi} \\
\ddot{\phi}
\end{array}\right]=\left[\begin{array}{lccc}
0 & 2 k_{m} k_{e}\left(M_{p} l r-I_{p}-M_{p} l^{2}\right) / R r^{2} \alpha & M_{p}^{2} g l^{2} / \alpha & 0 \\
0 & 0 & 1 \\
0 & 2 k_{m} k_{e}\left(R \beta-M_{p} l\right) / R r \alpha & M_{p} g l \beta / \alpha & 0
\end{array}\right]\left[\begin{array}{l}
x \\
\dot{x} \\
\varnothing \\
\dot{\phi}
\end{array}\right]+\left[\begin{array}{c}
0 \\
2 k_{m}\left(I_{p}+M_{p} l^{2}-M_{p} l r\right) / R r \alpha \\
0 \\
2 k_{m}\left(M_{p} l-r \beta\right) / R r \alpha
\end{array}\right] V_{a}} \\
& y=\left[\begin{array}{llll}
1 & 0 & 0 & 0 \\
0 & 0 & 1 & 0
\end{array}\right]\left[\begin{array}{c}
x \\
\dot{x} \\
\varnothing \\
\dot{\varnothing}
\end{array}\right]+\left[\begin{array}{l}
0 \\
0
\end{array}\right] V_{a}
\end{aligned}
$$

where $\alpha=\left[I_{p} \beta+2 M_{p} l^{2}\left(M_{w}+\frac{I_{w}}{r^{2}}\right)\right], \beta=\left[2 M_{w}+\frac{2 I_{w}}{r^{2}}+M_{p}\right]$

The mathematical model was programmed in Matlab for both the linearized and nonlinear model to verify how the systems behave and to design controllers for stabilizing the system. The obtained results shown in Figure 2 indicate the instability problem. This problem will be solved in the next sections.

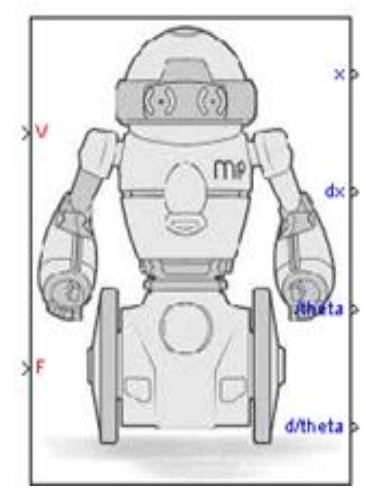

Figure 1. Simulink model of the TWBMR 

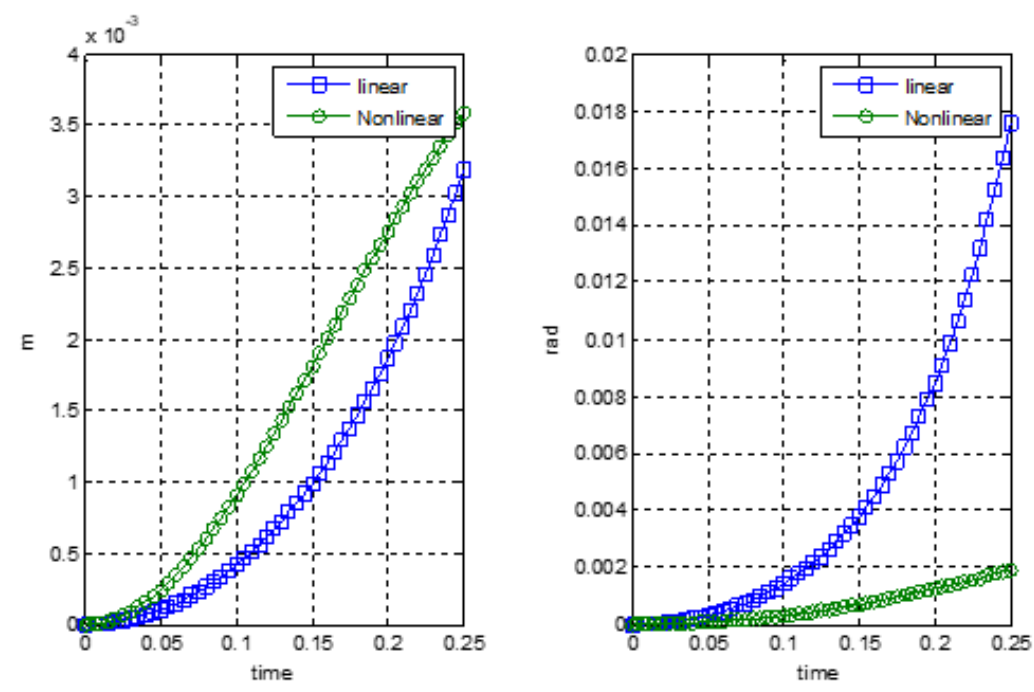

Figure 2. Position and angle of the nonlinear and linear system

\section{SLIDING MODE CONTROL MODEL}

The following general form gives the nonlinear system:

$$
x^{n}=f(x)+u
$$

where $\mathrm{n}$ denotes to the derivative order, $\mathrm{f}(\mathrm{x})$ represents the nonlinear functions with $\mathrm{x}$ is the vector of states to be controlled and $\mathrm{u}$ is the control signal generated by any of the considered controller. Generally speaking, the main control problem here is how to force the state vector $\mathrm{x}$ to follows the desired vector state $\mathrm{x}_{\mathrm{d}}$ under the influence of the model uncertainties and noise disturbance on the nonlinear system described by $\mathrm{f}(\mathrm{x})$. Where, the uncertainties can be considered as a system time delay on the system feedback states. The sliding mode controller generates two control signals, one is the hitting control signal which forces the system state to hit the sliding surface on which the desired state $x_{d}(t)$ is located and the equivalent control signal to slides the system state on the sliding surface until the desired state $x_{d}(t)$ is reached as shown in Figure 3 .

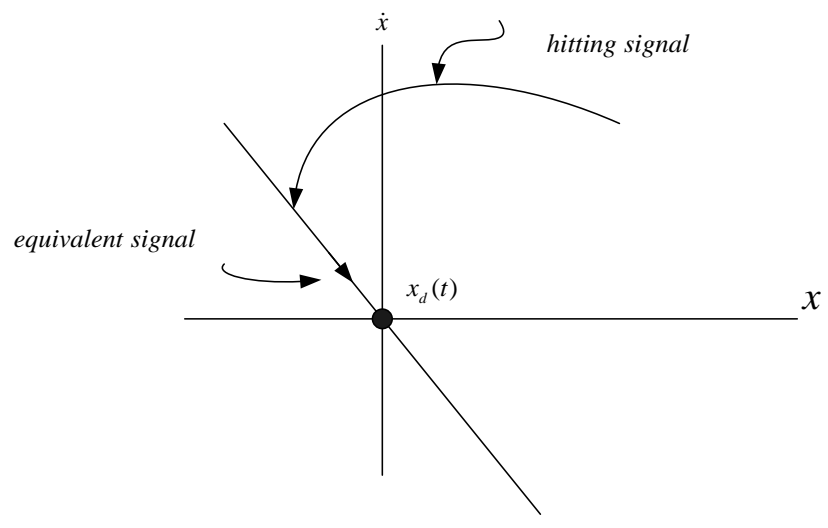

Figure 3. Sliding surface control signals

\subsection{Displacement sliding mode controller}

To force the trajectory of robot displacement $\mathrm{x}$ into the sliding surfaces $\mathrm{s}_{\mathrm{s}}$, let the sliding surface $\left(s_{s}=0\right)$ is defined by the following [11]:

$$
s_{s}=\left(\frac{d}{d t}+\lambda_{s}\right) e_{s}=0
$$


where, $\lambda_{s}$ is an adjustable positive parameter and $e_{s}$ is the error difference between the desired set point and the actual output displacement signal i.e

$$
e_{s}=x-x_{d}
$$

The sliding surface in (6) can be rewritten in the following form:

$$
s_{s}=\dot{e}_{s}+\lambda_{s} e_{s}=0
$$

The rate of change of the sliding surface $\left(\dot{s}_{S}=0\right)$ will be

$$
\dot{s}_{s}=\ddot{e}_{s}+\lambda_{s} \dot{e}_{s}=\ddot{x}-\ddot{x}_{d}+\lambda_{s} \dot{e}_{s}=0
$$

For the general nonlinear system model in (5) with derivative order $(n=2)$ can be rewritten as

$$
\ddot{x}=f_{1}\left(x, \theta_{p}\right)+u_{s}
$$

$\mathrm{u}_{\mathrm{s}}$ is the sliding control signal. Now in order to design this signal, the following Lyapunov function is considered $[12,13]$

$$
V_{s L}=0.5 s_{S}^{2}
$$

According to the Lyapunov stability concept, the squared distance to the sliding surface can be measured by the square of $s_{S}$ and to be decreased along all the system trajectories. Thereafter the following rate of change of the Lyapunov function is taken:

$$
\dot{V}_{s L} \leq-\eta_{s}\left|s_{s}\right|
$$

With $\eta_{s}$ is a small positive number near to zero. From (9) and (10), the following in-equality is obtained

$$
s_{s} \dot{s}_{s} \leq-\eta_{s}\left|s_{s}\right|
$$

By using the following equivalent in-equality relations [12]

$$
s_{s} \dot{s}_{s} \leq-\eta_{s}\left|s_{s}\right| \equiv \dot{s}_{s} \operatorname{sgn}\left(s_{s}\right) \leq-\eta_{s}
$$

The in equality in (14) with (9) and (10), can be manipulated to get

$$
\left[f_{1}\left(x, \theta_{p}\right)+u_{s}-\ddot{x}_{d}+\lambda_{s} \dot{e}_{s}\right] \operatorname{sgn}\left(s_{s}\right) \leq-\eta_{s}
$$

By inserting the model uncertainty $\Delta f_{1}\left(\mathrm{x}, \theta_{\mathrm{p}}\right)$ to get

$$
f_{1}\left(x, \theta_{p}\right)=\hat{f}_{1}\left(x, \theta_{p}\right)+\Delta f_{1}\left(x, \theta_{p}\right)
$$

Where $\hat{f}\left(\mathrm{x}, \theta_{\mathrm{p}}\right)$ is the estimate of $f\left(\mathrm{x}, \theta_{\mathrm{p}}\right)$, the in-equality given by (15) will be modified into

$$
\left[f_{1}\left(x, \theta_{p}\right)+\Delta \hat{f}_{1}\left(x, \theta_{p}\right)+u_{s}-\ddot{x}_{d}+\lambda_{s} \dot{e}_{s}\right] \operatorname{sgn}\left(s_{s}\right) \leq-\eta_{s}
$$

By inserting the upper bound $\mathrm{F}_{\mathrm{sd}}$ of the uncertainty $\Delta f_{1}\left(x, \theta_{p}\right)$ to get

$$
\left|\Delta f_{1}\left(x, \theta_{p}\right)\right|_{\max } \leq F_{s d}
$$

Then the in in-equality (17) can be rewritten as

$$
\begin{aligned}
& {\left[\hat{f}_{1}\left(x, \theta_{p}\right)+F_{s d}+u_{s}-\ddot{x}_{d}+\lambda_{s} \dot{e}_{s}\right] \operatorname{sgn}\left(s_{s}\right) \leq-\eta_{s}} \\
& {\left[\hat{f}_{1}\left(x, \theta_{p}\right)+F_{s d}-\ddot{x}_{d}+\lambda_{s} \dot{e}_{s}\right] \operatorname{sign}\left(s_{s}\right)+u_{s} \operatorname{sgn}\left(s_{s}\right) \leq-\eta_{s}}
\end{aligned}
$$


From (20), the following sliding control signal is obtained

$$
u_{s}=u_{e q}-k_{s d} \operatorname{sgn}\left(s_{s}\right)
$$

where, $u_{e q}$ is the equivalent control signal given by

$$
u_{e q x}=-\hat{f}_{1}\left(x, \theta_{p}\right)+\ddot{x}_{d}-\lambda_{s} \dot{e}_{s}
$$

With $\hat{f}_{1}\left(x, \theta_{p}\right)=-\frac{2 k_{e} k_{m}}{R r^{2}} \delta \dot{x}+M_{p} l \delta \dot{\theta}_{p}^{2} \sin \theta_{p}-F \delta-M_{p} l \delta \cos \theta_{p} \ddot{\theta_{p}}$ $k_{s d}=\frac{F_{s d}+\eta_{s}}{\left(\frac{2 k_{m}}{R r} \delta\right)}$ with $u_{s}=V_{a}$

The hitting control signal is represented by switching discontinuous term $k_{s d} \operatorname{sign}\left(s_{S}\right)$ with the sign function expressed by

$$
\operatorname{sgn}\left(s_{S}\right)=1 \text { if } s_{s}>0, \operatorname{sgn}\left(s_{s}\right)=0 \text { if } s_{s}=0 \text { and } \operatorname{sgn}\left(s_{S}\right)=-1 \text { if } s_{s}<0
$$

\subsection{Rotation sliding mode controller}

Similarly, to force the trajectory of robot angle rotation $\theta_{\mathrm{p}}$ into the sliding surface, assume the sliding surface $\left(s_{\theta}=0\right)$ is defined by

$$
s_{\theta}=\dot{e}_{\theta}+\lambda_{\theta} e_{\theta}=0
$$

width

$$
e_{\theta}=\theta_{p}-\theta_{d}
$$

The derivative of the sliding surface $\dot{s}_{\theta}$ is given by

$$
\dot{s}_{\theta}=\ddot{e}_{\theta}+\lambda_{\theta} \dot{e}_{\theta}=\ddot{\theta}-\ddot{\theta}_{d}+\lambda_{\theta} \dot{e}_{\theta}=0
$$

And due to limited space in this article, the same procedure taken in the previous design of displacement sliding mode controller are considered here with the following substitutions:

$$
\begin{aligned}
\eta_{s} & =\eta_{\theta}, u_{s}=u_{\theta}, F_{s d}=F_{\theta d}, \lambda_{s}=\lambda_{\theta}, k_{s d}=k_{\theta}, e_{s}=e_{\theta} \\
\ddot{\theta} & =f_{2}\left(x, \theta_{p}\right)+u_{\theta}
\end{aligned}
$$

And with Lyapunov function given by

$$
V_{\theta L}=0.5 s_{\theta}^{2}
$$

Similarly, the rate of change of Lyapunov function introduces the following in-equality:

$$
\begin{aligned}
& \dot{V}_{\theta L} \leq-\eta_{\theta}\left|s_{\theta}\right| \\
& s_{\theta} \dot{s}_{\theta} \leq-\eta_{\theta}\left|s_{\theta}\right|
\end{aligned}
$$

Accordingly, the sliding control signals for robot rotation control have been derived to get

$$
u_{\theta}=u_{e q_{\theta}}-k_{\theta} \operatorname{sgn}\left(s_{\theta}\right)
$$

Where the equivalent control signal $\mathrm{u}_{\mathrm{eq}_{\theta}}$ is represented by

$$
u_{e q_{\theta}}=-\hat{f}_{2}\left(x, \theta_{p}\right)+\ddot{\theta}_{d}-\lambda_{\theta} \dot{e}_{\theta}
$$

And the hitting control signal is given by $\mathrm{k}_{\theta} \operatorname{sign}\left(\mathrm{s}_{\theta}\right)$ with

$\hat{f}_{2}\left(x, \theta_{p}\right)=\frac{2 k_{e} k_{m}}{R r} \gamma \dot{x}-M_{p} g l \gamma \sin \theta_{p}-F Z \gamma \cos \theta_{p}-M_{p} l \gamma \ddot{x} \cos \theta_{p}$ 
$k_{\theta}=\frac{F_{\theta d}+\eta_{\theta}}{\left(-\frac{2 k_{m}}{R} \gamma\right)} \quad$ with $\quad u_{\theta}=V_{a}$

\subsection{Lyapunove stability analysis}

The design of the nonlinear controllers based on the Lyapunov design concept achieves the stability guarantee for TWBMR system model considered here. As known from the previous design of sliding mode controller, the sliding control signals for controlling the TWBMR displacement and tilt angle have been developed respectively based on the concept of the Lyapunov stability functions given by (12) and (27). And if these signals inserting respectively into (13) and (28), then by sequence the following two in-equalities are obtained:

$$
\begin{aligned}
& s_{s}\left(-k_{s d} \operatorname{sgn}\left(s_{s}\right)\right) \leq-\eta_{s}\left|s_{s}\right| \\
& s_{\theta}\left(-k_{s d} \operatorname{sgn}\left(s_{\theta}\right)\right) \leq-\eta_{\theta}\left|s_{\theta}\right|
\end{aligned}
$$

Consequently, because the constant parameters are always $\left(\eta_{s} \geq 0\right.$ and $\left.\eta_{\theta} \geq 0\right)$, so the related rate of change of Lyapunov functions denoted respectively by $\dot{V}_{\mathrm{SL}}$ and $\dot{\mathrm{V}}_{\theta \mathrm{L}}$ will be diminished to the value of less than or equal to zero. This will be demonstrated by the shown numerical simulation results later on.

\section{PID ALGORITHM BASED LQR WITH INTEGRAL CONTROL}

Here only $(x, \theta)$ are considered for the measurement. Two PID controllers are developed, one for controlling the tilte angle of the intermediate body (IB) for returning the body back to the upright position after a change caused by the disturbance force, and one for keeping the body wheels within a specified linear position from a specific reference position. In order to demonstrate the performance of the PID based LQR controller for locating the TWBMR to its desired position and angle, the collocated signals for the robot position and angle are feedback and compared to the desired position $\mathrm{x}_{\mathrm{d}}$ and desired angle $\theta_{\mathrm{d}}$ respectively. The position and angle errors are regulated through the following PID controllers represented respectively by $\mathrm{D}_{\mathrm{x}}(\mathrm{s})$ and $\mathrm{D}_{\mathrm{a}}(\mathrm{s})[10,14]$.

$$
D_{x}(s)=\frac{K_{D a} s^{2}+K_{p a} s+K_{i a}}{s} \text { and } D_{a}(s)=\frac{K_{D x} s^{2}+K_{p x} s+K_{i x}}{s}
$$

Accordingly the PID control signal can be described by the following equation:

$$
\begin{aligned}
& u=u_{P I D}(s)_{\text {position }}+u_{P I D}(s)_{\text {angle }} \\
& u=-D_{x}(s)\left[x(s)-x_{d}(s)\right]-D_{a}(s)\left[\theta(s)-\theta_{d}(s)\right]
\end{aligned}
$$

Since the pendulum angle and cart position dynamics are coupled to each other, and this makes the tuning tedious. The gain parameters of PID controllers determined by using of LQR and integral control with guaranteed desired response. The closed loop of the angle and position transfer functions after inserting the PID controllers in the coupled form shown in Figure 4 can be obtained as follows:

$$
\begin{aligned}
& G_{a}(s)=\frac{\theta(s)}{R_{a}(s)}=\frac{D_{a}(s) G_{a}(s)}{1+D_{a}(s) G_{a}(s)+D_{x}(s) G_{x}(s)} \text { and } \\
& G_{x}(s)=\frac{x(s)}{R_{x}(s)}=\frac{D_{x}(s) G_{x}(s)}{1+D_{a}(s) G_{a}(s)+D_{x}(s) G_{x}(s)}
\end{aligned}
$$

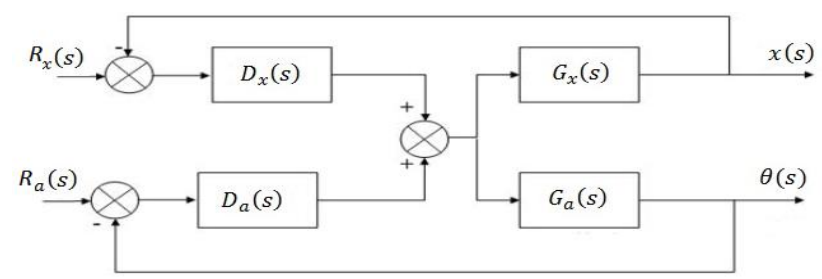

Figure 4. Parallel control double loop of TWBMR 
where the transfer functions $\mathrm{G}_{\mathrm{a}}(\mathrm{s})$ and $\mathrm{G}_{\mathrm{x}}(\mathrm{s})$ are derived by taking the Laplace transform operator on the state space model given by (3) and (4) to get [12]

$$
G_{a}(s)=\frac{a_{1}}{s^{4}+a_{2} s^{3}+a_{3} s^{2}+a_{4} s} \text { and } G_{x}(s)=\frac{b_{1} s^{2}+b_{2}}{s^{4}+b_{3} s^{3}+b_{4} 4 s^{2}+b_{5} s}
$$

And according to the given typical data in [12], the following identified transfer functions can be obtained:

$$
G_{a}(s)=\frac{0.2456}{s^{4}+0.0097 s^{3}-171.94 s^{2}-1.34 s} \text { and } G_{x}(s)=\frac{0.0815 s^{2}-11.2804}{s^{4}+0.0097 s^{3}-171.94 s^{2}-1.34 s}
$$

The $R_{a}(s)$ and $R_{x}(s)$ represent the command signals of the angle and position of the robot respectively. Then the close-loop transfer functions of the angle and position are expressed as follows:

$$
G_{a c}(s)=\frac{\theta(s)}{R_{a}(s)}=\frac{D_{a}(s) G_{a}(s)}{1+D_{a}(s) G_{a}(s)+D_{x}(s) G_{x}(s)} \text { and } G_{x c}(s)=\frac{x(s)}{R_{x}(s)}=\frac{D_{x}(s) G_{x}(s)}{1+D_{a}(s) G_{a}(s)+D_{x}(s) G_{x}(s)}
$$

The characteristic equation for the two-loop PID controller is given as below

$$
1+D_{a}(s) G_{a}(s)+D_{x}(s) G_{x}(s)=0
$$

Substituting the dynamics of $D_{a}(s), G_{a}(s), D_{x}(s)$ and $G_{x}(s)$ obtained previously, one obtains the following characteristic equation:

$1+\frac{K_{d a} s^{2}+K_{p a} s+K_{i a}}{s} \cdot \frac{a_{1}}{s^{4}+a_{2} s^{3}+a_{3} s^{2}+a_{4} s}+\frac{K_{d x} s^{2}+K_{p x} s+K_{i x}}{s} \cdot \frac{b_{1} s^{2}+b_{2}}{s^{4}+a_{2} s^{3}+a_{3} s^{2}+a_{4} s}=0$

Simplifying (42) yields the following equation

$$
\begin{gathered}
s^{5}+\left(a_{2}+b_{1} K_{d x}\right) s^{4}+\left(a_{3}+b_{1} K_{p x}\right) s^{3}+\left(a_{4}+a_{1} K_{d a}+b_{1} K_{i x}+b_{2} K_{d x}\right) s^{2} \\
+\left(a_{1} K_{p a}++b_{2} K_{p x}\right) s+\left(a_{1} K_{i a}+b_{2} K_{i x}\right)=0
\end{gathered}
$$

This will be compared to the following desired charterstic equation:

$$
\left(s-\beta_{1}\right)\left(s-\beta_{2}\right)\left(s-\beta_{3}\right)\left(s-\beta_{4}\right)\left(s-\beta_{5}\right)=0
$$

Where $\beta_{1}, \beta_{2}, \beta_{3}, \beta_{4}$ and $\beta_{5}$ are the desired eigen values.

In this section, we shall consider the design of stable control system based on desired specification. The twoloop PID controller's gains are formulated as the optimal state-feedback gains [12]. Comparing (43) and (44) gives

$$
\left[\begin{array}{cccccc}
0 & 0 & b_{1} & 0 & 0 & 0 \\
b_{1} & 0 & 0 & 0 & 0 & 0 \\
0 & b_{1} b_{2} & 0 & 0 & a_{1} \\
b_{2} & 0 & 0 & a_{1} & 0 & 0 \\
0 & b_{2} & 0 & 0 & a_{1} & 0
\end{array}\right]\left[\begin{array}{c}
K_{p x} \\
K_{i x} \\
K_{d x} \\
K_{p a} \\
K_{i a} \\
K_{d a}
\end{array}\right]=\left[\begin{array}{c}
p_{1}-a_{2} \\
p_{2}-a_{3} \\
p_{3}-a_{4} \\
p_{4} \\
p_{5}
\end{array}\right]
$$

But, there are six unknowns and five equations thus it is required to choose one variable arbitrarily. This will make the matrix in (45) invertible. It can be seen that the value of $\mathrm{K}_{\mathrm{ia}}$ can be chosen arbitrarily in (45). We get the following equations:

$$
\left[\begin{array}{c}
K_{p x} \\
K_{i x} \\
K_{d x} \\
K_{p a} \\
K_{d a}
\end{array}\right]=\left[\begin{array}{ccccc}
0 & 0 & b_{1} & 0 & 0 \\
b_{1} & 0 & 0 & 0 & 0 \\
0 & b_{1} & b_{2} & 0 & a_{1} \\
b_{2} & 0 & 0 & a_{1} & 0 \\
0 & b_{2} & 0 & 0 & 0
\end{array}\right]^{-1}\left[\begin{array}{c}
p_{1}-a_{2} \\
p_{2}-a_{3} \\
p_{3}-a_{4} \\
p_{4} \\
p_{5}-\left(a 1 * K_{i a}\right)
\end{array}\right]
$$

All the PIDx and PIDa controller parameters must be tuned simultaneously to achieve the best responses as desired. The gains of the PID controller are obtained using matlab program. 


\section{SIMULATION RESULTS}

The nonlinear model for the considered TWBMR system is simulated with the influence of the SMC and PID-LQR algorithm in order to improve the system performance under the nominal case and when the uncertainties due to effect of time delay and random noise on the robot feedback displacement and angle signals. The designed parameters of SMC and PID-LQR controllers are presented in Table 1. To assess the effectiveness of the proposed controllers computer simulations using MATLAB/SIMULINK are implemented.

Table 1. Design parameters of SMC and PID-LQR

\begin{tabular}{lcc}
\hline State & SMC & PID-LQR \\
\hline Displacement & $\lambda_{s}=8.186$ & $\mathrm{~K}_{\mathrm{px}}=300$ \\
& $k_{s d} 160.851$ & $\mathrm{~K}_{\mathrm{ix}}=50$ \\
Tilt angle & & $\mathrm{K}_{\mathrm{dx}}=20$ \\
& $\lambda_{\theta}=10.246$ & $\mathrm{~K}_{\mathrm{px}}=85.1$ \\
& $k_{\theta}=40.9$ & $\mathrm{~K}_{\mathrm{ix}}=30.25$ \\
& & $\mathrm{~K}_{\mathrm{dx}}=10.17$ \\
\hline
\end{tabular}

\subsection{Nominal case}

Under the lack of uncertainties due to time delay and disturbance noise, the considered system is judged by observing its position $(\mathrm{x})$, velocity $(\dot{x})$ tilt angle $(\theta)$ and angular velocity $(\dot{\theta})$ as shown in Figure 5 . It is seen that, both of controllers are successful to stabilize the system, where the SMC could achieve a better tracking performance in comparing to the PID- LQR where the settling time is lower for time response of the system outputs.
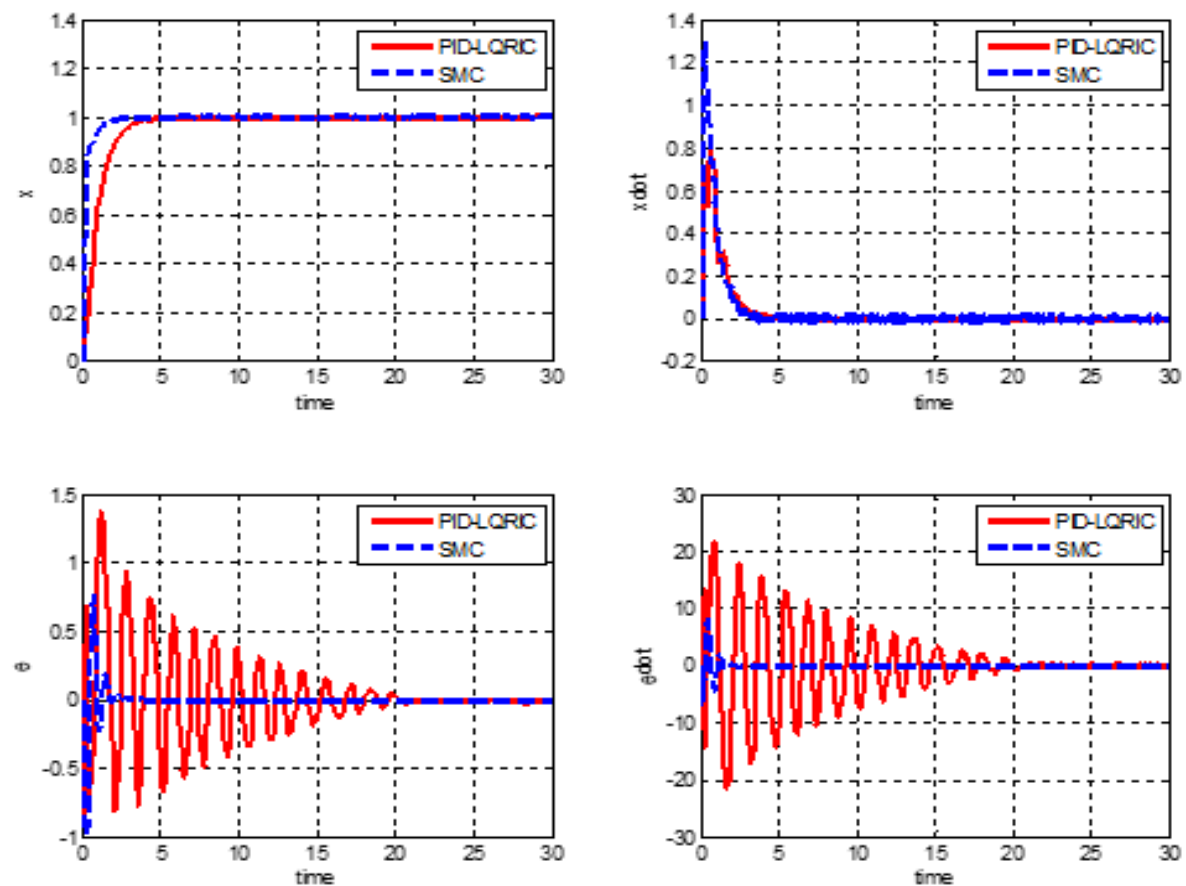

Figure 5. States of TWBMR using SMC and PID-LQR

Besides to the high tracking performance have been achieved by SMC, the system stability can be guaranteed as well, this can done by using of derived Lapunove function written in (32) and.(33) to get the Lyapunove function versus time for TWBMR system displacement and tilt angle as shown in Figure 6. Where, it is observed that the trajectories of these functions diminished to the values of less than or equal to zero which is the stability concept of the Lapunove theory. However, this stability guarantee could not be demonstrated graphically in case of design by PID-LQR. 

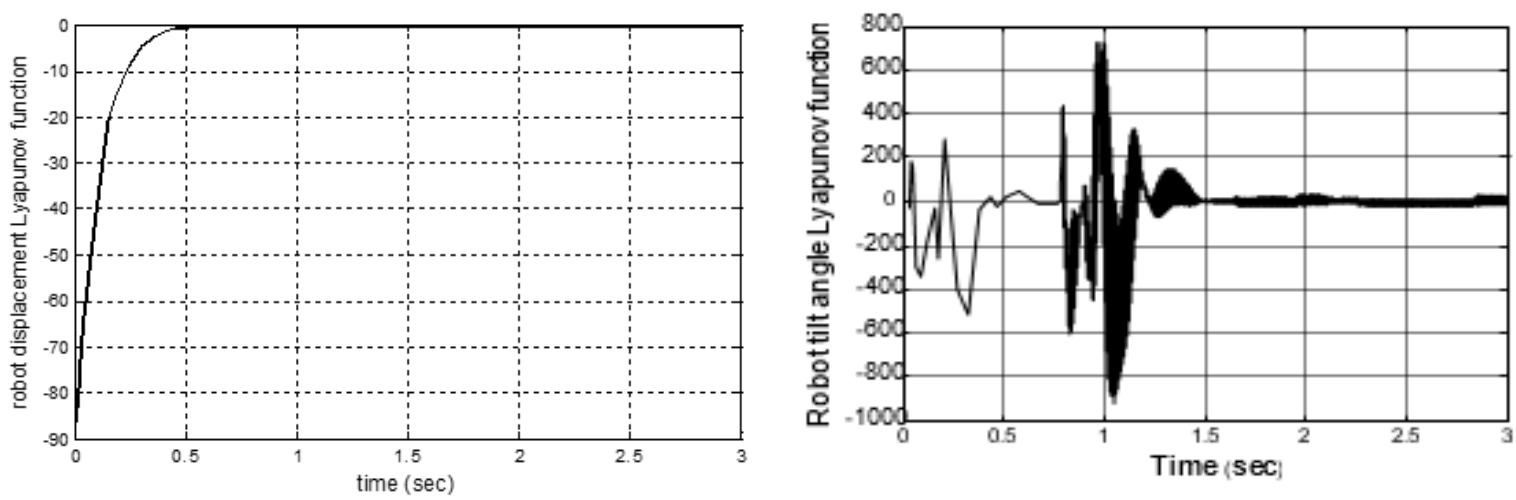

Figure 6. Lapunov function versus time for position and angle

\subsection{Uncertain time delay}

Time delay is a common phenomenon in robotic systems due to computational requirements and communication properties between or within high-level and low-level controllers as well as the physical constraints of the actuator and sensor. It is widely believed that delays are harmful for robotic systems in terms of stability and performance; therefore, in this paper, we discuss the influences of the displacement and tilt angle feedback delays on robotic system dynamics with each type of the considered controllers. In this simulation, the time delay with transfer function given as

$$
G_{D}(s)=\frac{-s+21.46}{s+21.46}
$$

In general, studies, such type of delay attempts to cause degradation in the system tracking performance. Figure 7 shows the influence of this uncertain model on the TWBMR system displacement and tilt angle. Where it is observed that, the SMC could keep the system stability with an acceptable performance while the un-stability is observed in case of PID-LQR due to the divergence of the displacement and tilt angle of the system.
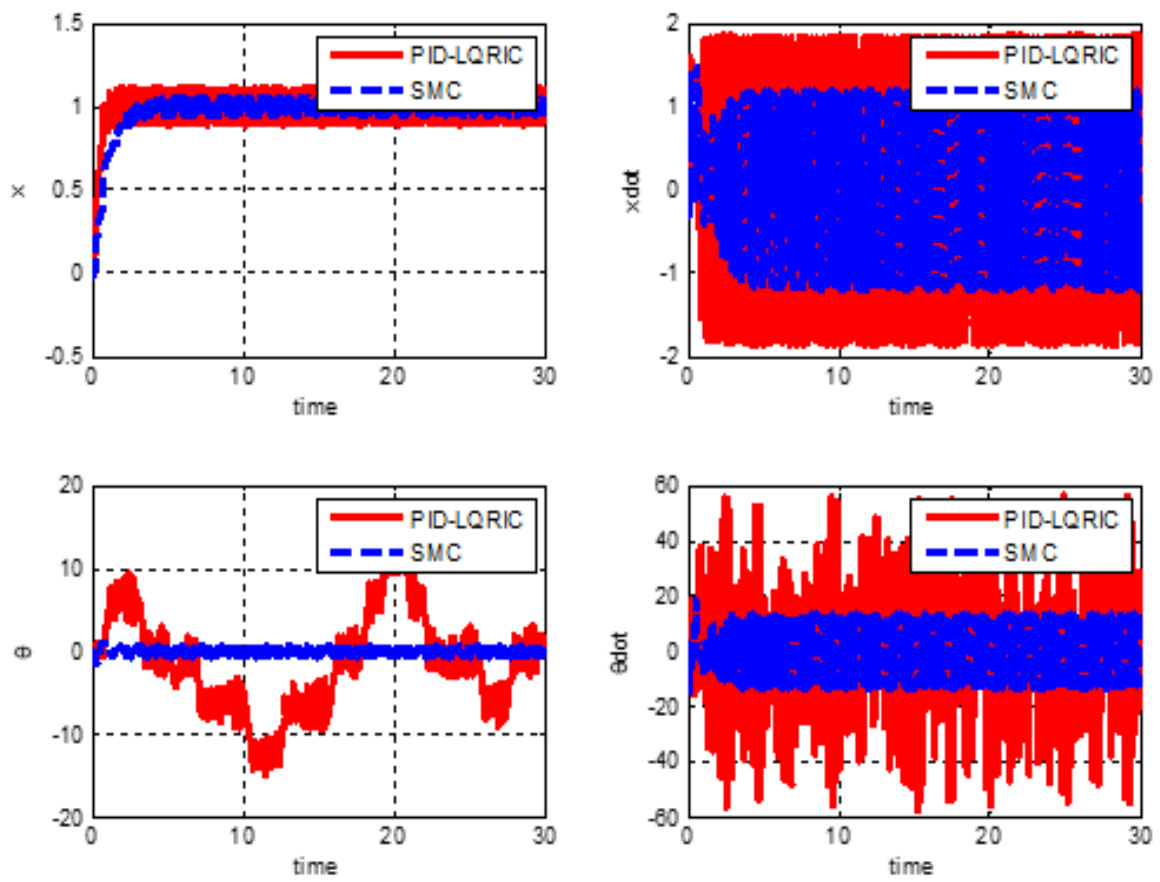

Figure 7. States of TWBMR with time delay 


\subsection{Uncertain random noise}

In addition, the uncertainty due to effect of noise is another factor has been analyzed here. In this simulation, the random noise is considered. Such type of noise is generated by using of simulink block called uniform random block with minimum and maximum interval \pm 0.5 . The influence of this signal on the system tracking performance can be seen from the time responses of the system as shown Figure 8 . Where it is observed that, the SMC suppresses the effect of this uncertain noise and keep a better tracking performance in comparing to controller design by PID-LQR.
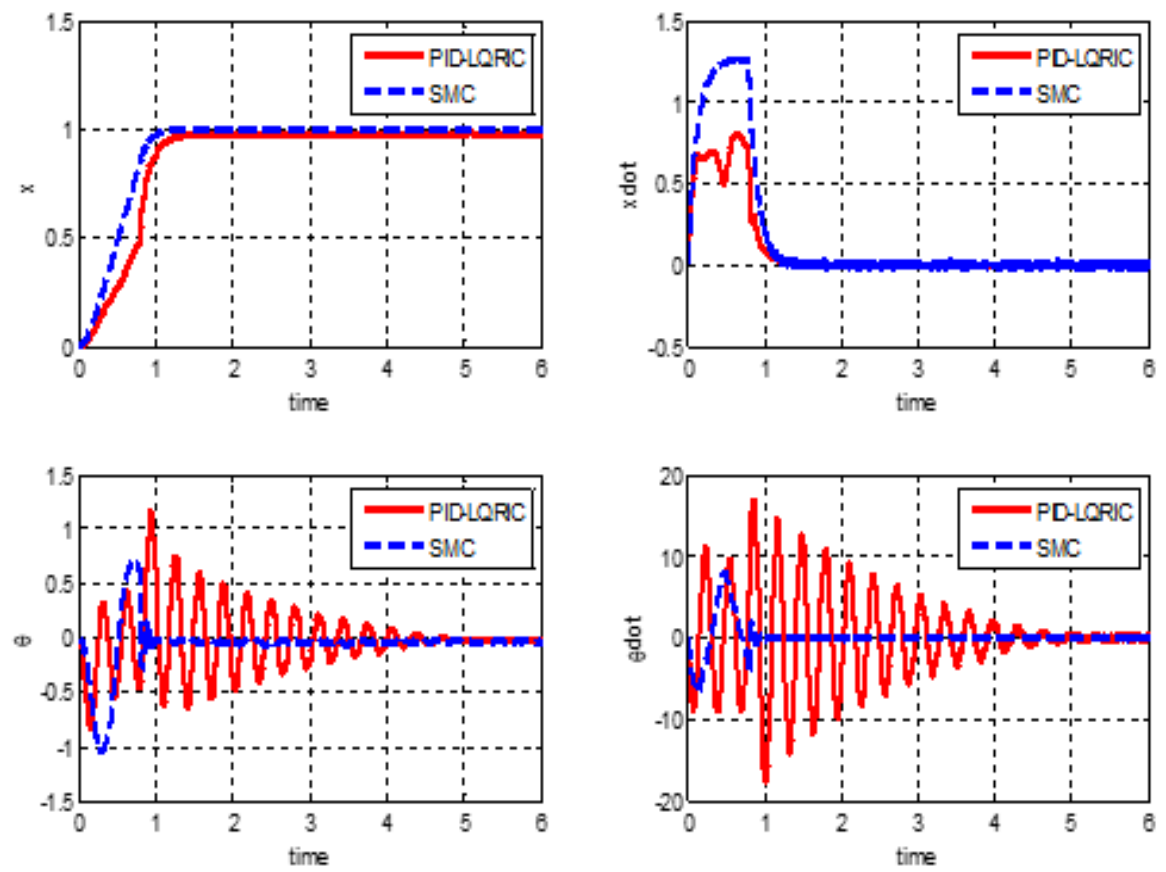

Figure 8. States of TWBMR with effect of noise

\section{CONLUSION}

The linear and nonlinear model for the TWBMR system was presented and simulated using Matlab program. In which the SMC and PID-LQR were investigated. The design of these two types of controllers have been done where in case of sliding mode controller, the two sliding mode control signals have been designed based on the stability concept of the Lyapunov theory, in order to force the system displacement and tilt angle with their derivatives to be sliding on the equivalent designed surfaces, until the desired set point is reached. Where in the other control designed approach, the parameters of PID-LQR for the double loop control have been designed based optimal state feedback control. The numerical simulation to test the effectiveness of each type of the controllers is carried out. Where in the nominal case, it is concluded that, both types of controllers achieve the input set point tracking with a better tracking performance was obtained when the SMC design technique is applied. In addition, the uncertainty due to effect of time delay dynamics and random noise signal is considered to test the robustness of the two control design approaches, and it is demonstrated that, the SMC is more robustness in comparing to the other type of the controller. This is because an acceptable tracking was achieved rather than other control design method in which the degradation in the tracking performance was observed while the random noise is applied, and the instability was occur when the time delay is considered.

\section{REFERENCES}

[1] J. Ahmed and Abougarair, "Model Reference Adaptive Control and Fuzzy Optimal Controller for Mobile Robotv," Journal of Multidisciplinary Engineering Science and Technology, vol. 6, no. 3, pp. 9722-9728, 2019.

[2] W. Junfeng and Z. Wanying, "Research on Control Method of Two-wheeled Self-balancing Robot," Fourth International Conference on Intelligent Computation Technology and Automation, vol. 1, pp. 476-479, 2011.

[3] K. Pathak, J. Franch, and S. K. Agrawal, "Velocity and Position Control of a Wheeled Inverted Pendulum by Partial Feedback Linearization," IEEE Trans. on Robotics, vol. 21, no. 3, pp. 505-513, 2005.

Robust control and optimized parallel control double loop design for mobile robot (Ahmed J. Abougarair) 
[4] F. Grasser, et al., "A mobile inverted pendulum," IEEE Transactions on Industrial Electronics, vol. 49, no. 1, pp. 107-114, 2002.

[5] Zinober, "Deterministic control of uncertain systems," London: Peter Peregrinus Press, 1990.

[6] L. C. McNinch and H. Ashrafiuon, "Predictive and Sliding Mode Cascade Control for Unmanned Surface Vessels," IEEE American Control Conference, pp. 184-189, 2011.

[7] A. Mahini, et al., "An experimental setup for autonomous operation of surface vessels in rough seas," Robotica, vol. 31, no. 5, pp. 703-715, 2013.

[8] L. B. Xie, et al, "Approximated modeling and sliding mode control for systems with multiple time delays," Institute of Electrical and Electronics Engineers, pp. 3149-3154, 2012.

[9] M. C. Pai, "Robust tracking and model following for uncertain time-delay systems with input nonlinearity," Journal complexity, vol. 21, no. 2, pp. 66-73, 2015.

[10] Y. Gong and X. Wu., "Research on Control Strategy of Two-wheeled Self-balancing Robot," International Conference on Computer Science and Mechanical Automation, pp. 281-284, 2015.

[11] L. Li, et al, "Dynamic Model and Balance Control of Two-wheeled Robot with Non-holonomic Constraints," Proceeding of the 11th World Congress on Intelligent Control and Automation Shenyang, pp. 503-508, 2014.

[12] A. J. Abougarair, "Balancing and trajectory tracking control of mobile robot using robust intelligent system based on 3D Animation," Ph. D dissertation, Alneelain University, 2018.

[13] A. Elmelhi, "Flight Vehical Stabilization Based Fuzzy Logic Control Design and Optimization," $a$ Ph. $D$ dissertation, Beihang University, 2005.

[14] S. Li, "Applied Nonlinear Control," Prentice-Hall, Inc. U.S.A, 1991. 